\title{
Conditions for the Optimality of the Averaged Problem of Nonlinear Programming and Synthesis of the Philippov's Theorem on Speed of Motion of Systems with the Discontinued Right Part of Differential Equations
}

\author{
${ }^{1}$ Margarita A. ZAEVA, ${ }^{2}$ Anatoly M. TSIRLIN
}

${ }^{1}$ National Research Nuclear University MEPhI, 115409, Russian Federation, Moscow, Kashirskoe shosse, 31 ${ }^{2}$ Ailamazyan Program Systems Institute of the RAS, 152020, Russian Federation, Yaroslavl region, Pereslavl district, Veskovo village, Petra Pervogo st., 4A MAZayeva@mephi.ru

Abstract: A generalization of the Filippov's velocity averaging method in the problem of determining the slip velocity of a dynamical system with discontinuous right-hand sides is proposed. It is shown that in the multidimensional case such an extension is reduced to the solution of the averaged nonlinear programming problem and the methods of averaged optimization can be used to calculate the slip velocity. Problems with various dimensions of the vector of admissible velocities and the number of surfaces of control's discontinuity are considered.

Keywords: discontinuous systems, sliding regimes, slip velocity, averaged optimization.

\section{INTRODUCTION}

Sliding modes in systems described by ordinary differential equations (ODEs) are the subject of many and many years of research.A brief survey of the problems of which is contained in [1], an incentive to write this article.

Formulation of the problem. We consider a dynamical system

$$
\dot{x}=f(x, t, u), \quad x, f \in R^{n}, \quad u \in V_{u} \supset R^{k} .
$$

Control $u$ and continuous function $f$ of the argument $u$ has a discontinuity on surfaces $S$ characterized by conditions

$$
s_{i}(x)=0, \quad i=1, \ldots, m, \quad m \leq n-1,
$$

so that

$$
\begin{aligned}
& f(x, t, u)=f_{+}^{i}(x, t, u), \quad \text { if } \quad s_{i}>0, \\
& f(x, t, u)=f_{-}^{i}(x, t, u), \quad \text { if } \quad s_{i}<0, \quad i=1, \ldots, m .
\end{aligned}
$$

Vector-valued functions $f_{+}^{i}, f_{-}^{i}$ with components $f_{j+}^{i}, f_{j-}^{i}, j=1, \ldots, n$, are differentiable in $x$ and $t$. For a fixed $u$ the solution of the equations (1) for any of them exists and is unique. If on the surface of control's discontinuity for all $t>0$ and $x \in S$ the following conditions of attraction are satisfied, for example, of the form:

$$
\begin{aligned}
& \left(\frac{d s_{i}}{d t}\right)_{+}=\varphi_{+}^{i}(x, u, t)=\sum_{j=1}^{n} \frac{\partial s_{i}}{\partial x_{j}} f_{j+}^{i}(x, t, u)+\frac{\partial s_{i}}{\partial t}<0, \\
& \left(\frac{d s_{i}}{d t}\right)_{-}=\varphi_{-}^{i}(x, u, t)=\sum_{j=1}^{n} \frac{\partial s_{i}}{\partial x_{j}} f_{j-}^{i}(x, t, u)+\frac{\partial s_{i}}{\partial t}>0, \quad i=1, \ldots, m,
\end{aligned}
$$


Conditions for the Optimality of the Averaged Problem of Nonlinear Programming and Synthesis of the Philippov's Theorem on Speed of Motion of Systems with the Discontinued Right Part of Differential Equations

then the system, being on the switching surface, moves along this surface in the control switching mode with a high frequency (sliding mode). In this case, the switching surface is called the sliding surface.

The conditions of attraction in the form (4) are sufficient, but not necessary for the appearance of a sliding mode. The sliding mode can arise on the intersection of the surfaces $s_{i}(x, t)=0$, and for its emergence it is required that the system, by virtue of the equations of motion, approach the origin of coordinates in space, along the axes of which the functions $s_{i}(x, t)$, with nonzero velocity. An example of such a situation is given in [2].

The task set by numerous researchers[3]-[6], was to find the equation the motion of the system along the surface (2) is subject to:

$$
\dot{x}=f_{s m}(x, t), \quad x(0) \in S .
$$

The equation is called the slip equation.

In for the case of a single discontinuity surface $(m=1)$ it is suggested to find the slip velocity $f_{s m}(x, t)$ for each value $x$ and $t$ as an element of a convex hull spanned by vectors $f_{+}(x, t)$ and $f_{-}(x, t)$ and satisfying sliding conditions along the discontinuity surface:

$$
\begin{gathered}
\frac{d s}{d t}=f_{s}(x, t)=\sum_{j=1}^{n} \frac{\partial s}{\partial x_{j}} f_{s m}^{j}(x, t)+\frac{\partial s}{\partial t}=0, \\
f_{s m}(x, t)=\mu_{+} f_{+}(x, t)+\mu_{-} f_{-}(x, t), \quad \mu_{+}+\mu_{-}=1, \quad \mu_{+} \geq 0, \quad \mu_{-} \geq 0 .
\end{gathered}
$$

In other words, the slip velocity vector is found as the weighted-average value of the vectors of admissible velocities $f_{+}(x, t)$ и $f_{-}(x, t)$ with weight coefficients chosen so that $f_{s m}(x, t)$ touched the sliding surface.

Thus, the additional definition of A.F. Filippov from [3] ois based on averaging the admissible speeds of the system. Systems with two vectors of admissible velocities and one sliding surface are considered [3].

In the general case, the number of permissible velocities can be different, as is the number of sliding surfaces. The surfaces $s_{i}(x)=0$ partition the phase space of the system (1) into $2^{m}$ subspaces $x_{r}, r=1, \ldots, 2^{m}$, in each of which its some combination of signs $s_{i}$, and hence, in each of which there can be it's its velocity vector $f(x, u, t)$. The problem arises of determining how many and which of the admissible velocities need to be averaged and how to choose the weight factors.

In [2] it is said: «'As for the vector case, the author does not know the works in which procedures would be proposed that allow in an explicit form to write out the slip equations over the intersection of the discontinuity surface.»'.

We denote the set of vectors of admissible velocities by $F(x, u, t)$. The number of these vectors for fixed values of $x \in S$ and $t$ can be less than and greater than $2^{m}$. It is only important that in each subspace $x_{r}$ the conditions of attraction to the intersection of sliding surfaces be satisfied.

We will follow the method of averaging the admissible velocities of AF. Filippov, to determine the slip velocity as the average velocity of the system along such a direction, for which the average rates of change of the functions $s_{i}(x)$ are zero (see [1]).

In order to obtain algorithms for determining the slip velocity, it is necessary to formalize this definition for the case when the sliding surfaces are several and the set of permissible velocities is given in various forms. With this formalization method, the problem of determining the slip velocity by the method of averaging the admissible velocities turns out to be the averaged problem of nonlinear programming. This allows us to find the slip velocity $f_{s m}(x, t)$ using the averaged optimization [7]. 
Conditions for the Optimality of the Averaged Problem of Nonlinear Programming and Synthesis of the Philippov's Theorem on Speed of Motion of Systems with the Discontinued Right Part of Differential Equations

Before we formalize the problem of determining the slip velocity and expound the algorithms for solving it, we recall the optimality conditions for the averaged nonlinear programming problem.

\section{Solving the Problems of Averaged Optimization}

The averaged nonlinear programming problem has the form

$$
\overline{f_{0}(u)} \rightarrow \max _{p(u)} / \overline{f_{i}(u)}=0, \quad i=1, \ldots, m, \quad u \in V_{u} .
$$

Here

$$
\overline{f_{i}(u)}=\int_{V_{u}} f_{i}(u) P(u) d u, \quad i=0, \ldots, m .
$$

The functions $f_{i}(u)$ are continuous and bounded on the set $V_{w}$ and this set is a probability measure that determines the weight of each of the ordinates of the function $f_{i}(u)$ when its averaging.

The following assertions are true:

1. The optimal solution of the problem ((7)) - the probability measure $P^{*}(u)$ is concentrated at discrete points whose number does not exceed $m+1$, so that

$$
P^{*}(u)=\sum_{v=0}^{m} \mu_{v} \delta\left(u-u^{v}\right),
$$

where $\delta-$ is the Dirac delta function. $u^{v}$ - are called the base values of the vector $u$, and $\mu_{v}-$ are nonnegative weighting coefficients whose sum is equal to one.

2. There exists a nonzero vector $\lambda$ with components $\lambda_{i}, i=0,1, \ldots, m$, such that the base values of the vector $u$ are solutions of the minimax problem

$$
L(\lambda, u)=\sum_{i=0}^{m} \lambda_{i} f_{i}(u) \rightarrow \operatorname{minmax}_{\lambda} \operatorname{maV}_{u}
$$

On an optimal solution, the values of the Lagrange function $L\left(\lambda^{*}, u^{v}\right)$ Mare maximal and the same for all $v=0,1, \ldots, m$.

We emphasize that the existence of Lagrange multipliers does not require the smoothness of the functions $f_{i}(u)$. It is enough that they are continuous and limited. The set $V_{u}$ can also consist of isolated isolated points.

3. The weighting factors $\mu_{v} \geq 0$ corresponding to the base values $u^{v}$ satisfy the system of linear equations

$$
\sum_{v=0}^{m} \mu_{v} f_{i}\left(u^{v}\right)=0, \quad \sum_{v=0}^{m} \mu_{v}=1, \quad i=1, \ldots, m \text {. }
$$

The existence of a nonnegative solution of this system corresponds to the fact that in the $m$-dimensional space with coordinates $f_{i}$ the origin of coordinates belongs to the convex hull of the set $F$ of the vectors $f^{v}=f\left(u^{v}\right)$. $\mathrm{f}$ the number of base values of $u$ is less then $m+1$, then the origin in this space lies on the boundary of the convex hull $F$.

Thus, the solution of the averaged problem consists of two stages. On the first the most time-consuming stage,we find the basic values $u=u^{v}$ from the solution of the minimax problem (10), in the second stage, at fixed $u^{v}$ we find the weight coefficients $\mu_{v}$ by the conditions (11) .

In a number of problems, the number of base values of $u$ always to be less than $m+1$. In particular, if the set $V_{v}$ can be partitioned into $r<m+1$ subsets so that for each of them the problem (10) has a unique solution $u^{v}$, for example, each subset strictly convex, then $v$ changes from one to $r$ (see [8] ). In this case, on each subset, the optimality conditions of the convex problem are written, and the vector $\lambda$ is found by the condition that the values of the Lagrangian are equal in the maxima found. 
Conditions for the Optimality of the Averaged Problem of Nonlinear Programming and Synthesis of the Philippov's Theorem on Speed of Motion of Systems with the Discontinued Right Part of Differential Equations

We emphasize that the number of base values of $u$ is not related to the dimension of this vector, but depends only on the number of average conditions $m$ in the problem (7). In this case, the set of basic values of $u^{v}$ may not be unique (see example 2).

If in the problem (7) instead of the requirement of maximum the requirement of minimum appears, then in (10) $\max$ and $\min$ are interchanged.

\section{Degenerate Case}

As in the problem of non-linear programming, in the averaged problem (7) addition to regular solutions, degenerate solutions can also exist. For degenerate solutions, the factor $\lambda_{0}$ in the Lagrange function is zero, which corresponds to the case when the constraint system completely determines the solution, and consequently, the target function $f_{0}$ does not affect it. Such a situation, in particular, takes place when the set $V_{u}(x, t)$ consists of discrete values of the vector $u$ and the number of these values does not exceed $m+1$. In this case the problem (4) is degenerate, in the Lagrange function $L(\lambda, u)$ the factor $\lambda_{0}=0$ and all the admissible values $u$ are basic. The solution of the problem reduces to determining the weight coefficients $\mu_{v}$ from the system of linear equations (11).

When the number of admissible values of $u \in V_{u}$ is equal to $K<m+1$, then the equations have no solutions in the general case. But from the number of $m$ functions $f_{i}(u)$ we can choose $K-1$ functions whose average values are zero (there exists a nonnegative solution of the equations (11) with respect to $K$ weighting coefficients).

If the number of admissible values of $u \in V_{u}$ is greater than $m+1$, then the possible slip speed is not unique. Each of them has its own set of basic permissible speeds and weight coefficients. The choice of the slip velocity in this case is carried out according to the conditions (10) taking into account the objective function $f_{0}$. The form of this objective function is determined by the specific content of the task. But in any case it contains the operation of averaging the permissible velocities. In particular, the criterion of optimality can be the maximum of the average rate of change of some function $F_{0}$ from state variables along a slip trajectory or the maximum of projection of the slip velocity on the direction of the selected vector $\gamma$. The last statement corresponds to the case when the function $F_{0}$ is linear.

\section{The Task of Additional Determining the Speed of Sliding}

\section{Formulation of the Averaged Problem}

Average on the set of permissible velocities $V_{u}(x, t)$ is the rate of change of the state vector

$$
\overline{f(x, u, t)}^{u}=\int_{V_{u}} f(x, u, t) P(u, x, t) d u .
$$

The choice of the measure $P(u, x, t)$ is bounded by the condition that the mean value of the rate of change of each of the functions $s_{i}(x, t)$, equal to zero, the intersection of which forms a sliding surface, by the equations (1):

$$
{\overline{\varphi^{i}(x, u, t)}}^{u}=\int_{V_{u}} \varphi^{i}(x, u, t) P(u, x, t) d u=0 \quad i=1, \ldots, m .
$$

Here

$$
\varphi^{i}(x, u, t)=\sum_{j=1}^{n} \frac{\partial s_{i}}{\partial x_{j}} f_{j}(x, u, t)+\frac{\partial s_{i}(x, t)}{\partial t} .
$$

When averaging in this expression, the functions $f_{j}(x, u, t)$ are replaced by the functions ${\overline{f_{j}(x, u, t)}}^{u}$. 
Conditions for the Optimality of the Averaged Problem of Nonlinear Programming and Synthesis of the Philippov's Theorem on Speed of Motion of Systems with the Discontinued Right Part of Differential Equations

In addition to the conditions the required solution $P(u, x, t)$ satisfies the following requirements:

$$
\int_{V_{u}} P(u, x, t) d u=1, \quad P(u, x, t) \geq 0 \quad \forall u \in V_{u} .
$$

The problem of determining the maximum mean velocity of moving of the system in the direction of the vector $\gamma=\left(\gamma_{1}, \ldots, \gamma_{n}\right)$, normalized so that $\sum_{j=1}^{n} \gamma_{j}^{2}=1$, and tangent to the slip surface, provided that the average rates of change of the functions $s_{i}(x, t)$ are zero, takes the form

$$
\sum_{j=1}^{n} \gamma_{j}{\overline{f_{j}(x, t, u)}}^{u} \rightarrow \max ,
$$

under conditions

$$
\sum_{j=1}^{n}\left(\frac{\partial s_{i}}{\partial x_{j}}{\overline{f_{j}}(x, t, u)}^{u}+\frac{\partial s_{i}}{\partial t}\right)=0, \quad i=1, \ldots, m .
$$

Here averaging over $u$ s performed on the set $V_{u}$ of admissible values of control. Since the motion occurs along the intersection of the switching surfaces, the vector $\gamma \in R^{n}$ must satisfy the requirements:

If $n>m+1$, then this vector is not unique.

$$
\left(\gamma^{T}, \frac{\partial s_{i}}{\partial x}\right)=0, \quad i=1, \ldots, m \text {. }
$$

The set of admissible velocities can be determined by conditions of the next type

$$
f(x, t, u)=\left\{\begin{array}{ll}
f_{+}(x, t, u) & \mathrm{乃} \\
( &
\end{array}(x, t)<0 .\right.
$$

The number of admissible velocities in this case is arbitrarily large.

The velocity of the system in the sliding mode $f_{s m}(x, t)$ is the average velocity of the system on the optimal solution of the problem (16), (17).

\section{Optimality Conditions of the Solution of the Averaged Problem}

The additional determination of the slip velocity requires the calculation of the base values of control $u^{v}(x, t)$ and the corresponding velocity vectors $f^{v}(x, t)=f\left(x, t, u^{v}\right)$ from the averaged solution of the non-linear programming problem (16), (17). The number of these base values does not exceed $m+1$.They correspond to a minimum in $\lambda$ of the maximum in the $u$ Lagrange function

$$
L=\sum_{j=1}^{n} \gamma_{j} f_{j}(x, t, u)+\sum_{i=1}^{m} \lambda_{i}\left(\sum_{j=1}^{n} \frac{\partial s_{i}}{\partial x_{j}} f_{j}(x, t, u)+\frac{\partial s_{i}}{\partial t}\right) \rightarrow \min _{\lambda} \max _{u}
$$

In this problem, the value $\lambda_{0}$ is not zero, which means that it can be considered equal to one. On the optimal solution for the base values of the velocity vector $f^{v}=f\left(x, t, u^{v}\right)$ expression

$$
L^{*}=\sum_{j=1}^{n} \gamma_{j} f_{j}\left(x, t, u^{v}\right)+\sum_{i=1}^{m} \lambda_{i}^{*}\left(\sum_{j=1}^{n} \frac{\partial s_{i}}{\partial x_{j}} f_{j}\left(x, t, u^{v}\right)+\frac{\partial s_{i}}{\partial t}\right)
$$

maximal, and therefore, the same for all values of $v=0, \ldots, m$. It is clear that the base velocities $f^{v}$, which are the solution of the averaged problem (16), (17), are different for different directions $\gamma$.

The system in sliding mode is characterized by equations

where

$$
\dot{x}_{s}=f_{s m}(x, t), \quad x_{s}(0) \in S,
$$

$$
f_{s m}(x, t)=\sum_{v=0}^{m} \mu_{v} f^{v}(x, t), \quad x_{s}(0) \in S .
$$

The weight coefficients $\mu_{v}$ are found from equations

$$
\sum_{j=1}^{n} \frac{\partial s_{i}}{\partial x_{j}} \sum_{v=0}^{m} \mu_{v} f_{j}\left(x, t, u^{v}\right)+\frac{\partial s_{i}}{\partial t}=0, \quad i=1, \ldots, m, \quad \sum_{v=0}^{m} \mu_{v}=1, \quad \mu_{v} \geq 0 .
$$


Conditions for the Optimality of the Averaged Problem of Nonlinear Programming and Synthesis of the Philippov's Theorem on Speed of Motion of Systems with the Discontinued Right Part of Differential Equations

The figure 1 illustrates this case.

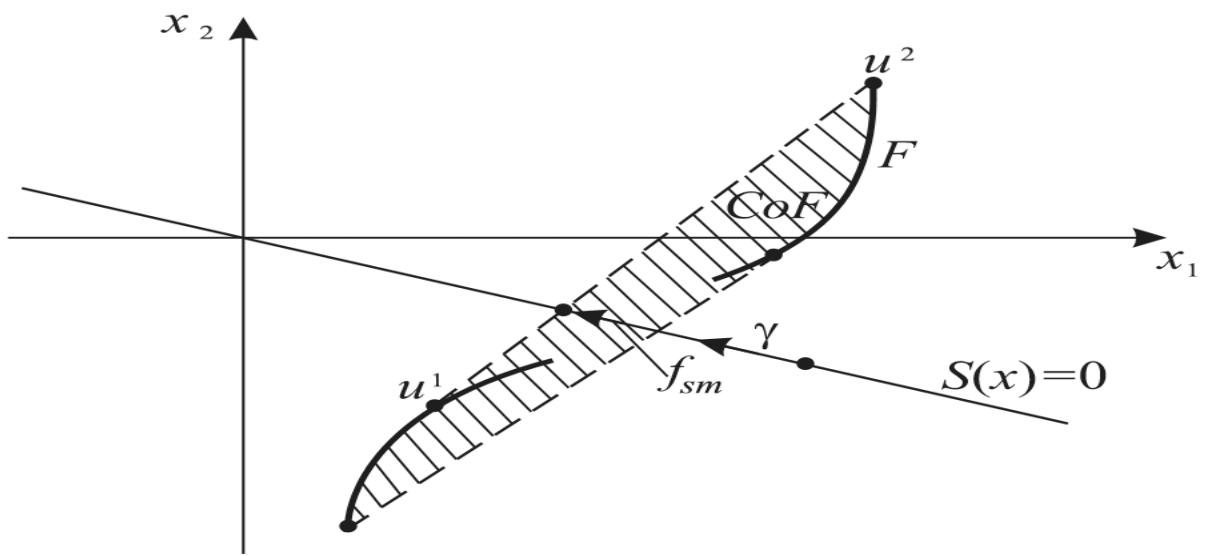

Fig1. The additional determination in the speed of sliding for the case when the set of permissible speeds depends on the control.

The averaged problem (16), (17) is degenerate if the number of vectors of admissible velocities does not exceed $m+1$ In this case, all these vectors are basic and it is only necessary to solve the problem of calculating the weight coefficients $\mu_{v}$ y the conditions (23).

When the number of admissible velocity vectors $K$ is less than $m+1$, the number of sliding surfaces does not exceed $K-1$. And the problem is degenerate. For example, these vectors can be two $f^{+}(x, t)$ and $f^{-}(x, t)$, and switching surfaces $-s_{i}(x)=0$ are greater than one. In this case, the switching between the vectors $f^{+}$ and $f^{-}$is determined by the sign of only one of the functions $s_{1}(x, t)$, and the conditions of attraction to other sliding surfaces are provided by the dynamic properties of the system. The surface $s_{1}(x, t)=0$ is a sliding surface. Slip velocity vector

$$
f_{s m}(x, t)=\mu_{0}(x, t) f^{+}(x, t)+\mu_{1}(x, t) f^{-}(x, t),
$$

where $\mu_{0}$ and $\mu_{1}$ are determined from the conditions ((23)) for $i=1$ when summing over $K$ from zero to one.

Let's illustrate by examples the use of additional definition, based on averaging of permissible speeds. It coincides with the additional definition of A.F. Filippov from for the considered case of motion along the switching line on a two-dimensional phase plane with two admissible velocities.

\section{SOME EXAMPLES}

The simplest way is to find the slip velocity in the case when the averaged problem turns out to be degenerate, since in this case all admissible controls are basic and the problem reduces to the calculation of the weight coefficients. These are the examples most often considered in publications. Let us show that for such cases the introduced general additional definition of the slip velocity by the method of averaging the admissible velocities leads to known results. Then we consider the more general case when the averaged problem is nondegenerate. In this case, its solution allows us to find the slip velocity without any geometric constructions.

Example1. A degenerate problem. Linear second-order system with relay control: [1], [3]

$$
\begin{aligned}
& \dot{x}_{1}=x_{2}, \quad \dot{x}_{2}=u, \\
& u=-u_{0} \operatorname{sign}(s), \quad s=c x_{1}+x_{2}, \\
& u_{0}, c>0 .
\end{aligned}
$$


Conditions for the Optimality of the Averaged Problem of Nonlinear Programming and Synthesis of the Philippov's Theorem on Speed of Motion of Systems with the Discontinued Right Part of Differential Equations

For this system, the conditions of attraction ((4)) to the slip line $s\left(x_{1}, x_{2}\right)=0, m=1$ are fulfilled. The set $V_{u}$ consists of two, i.e. from $m+1$, elements, so that the averaged problem (16), (17) is degenerate, and the two allowable velocity vectors are basic. To solve the averaged nonlinear programming problem, we need to find the weighting factors $\mu_{v}$ corresponding to the basic controls. The equations of sliding have the form:

$$
\begin{aligned}
& \dot{x}_{1}=x_{2}, \\
& \dot{x}_{2}=f_{2 s m}(x)=u_{0}[\mu-(1-\mu)]=-u_{0}(2 \mu-1) .
\end{aligned}
$$

The factor $\mu$ is determined by the condition that the mean rate of change is equal zero $s\left(x_{1}, x_{2}\right)$

$$
\dot{s}=c x_{2}+f_{2 s m}\left(x_{1}, x_{2}\right)=0 \text {, }
$$

where from

since $\mu<1$, then $c x_{2}<u_{0}$.

$$
\mu=0,5\left(\frac{c x_{2}}{u_{0}}+1\right) \cdot \mu=0,5\left(\frac{c x_{2}}{u_{0}}+1\right) .
$$

After substitution in (26) the slip equations will take the form:

which corresponds to the known result.

$$
\begin{aligned}
& \dot{x}_{1}=x_{2} \\
& \dot{x}_{2}=-c x_{2},
\end{aligned}
$$

Example 2. Nondegenerate problems.

A. Third order system [1]:

$$
\begin{array}{ll}
\dot{x}_{1}=u_{1}, & u_{1}=-\operatorname{sign}\left(x_{1}\right), \\
\dot{x}_{2}=u_{2}, & u_{2}=-\operatorname{sign}\left(x_{2}\right) . \\
\dot{x}_{3}=u_{1} u_{2} . &
\end{array}
$$

In this case, $m=2\left(s_{1}=x_{1}, s_{2}=x_{2}\right)$, and the number of vectors of admissible speeds $K=4$, i.e. more than $m+1$. Permissible speeds $f\left(u_{1}, u_{2}, u_{1} u_{2}\right)$ :

$$
\begin{gathered}
f^{1}=(1 ; 1 ; 1) ; \quad f^{2}=(1 ;-1 ;-1) ; \\
f^{3}=(-1 ; 1 ;-1) ; \quad f^{4}=(-1 ;-1 ; 1) .
\end{gathered}
$$

We denote their projections on the coordinate axes by $f_{j}^{k}, \quad j=1,2,3$.

To determine the slip velocity, we need to select the vector $\gamma$ and solve the averaged nonlinear programming problem (16), (17), by finding three basic vectors of admissible velocities.

As a vector $\gamma$ we select a unit vector that coincides in direction with the axis $x_{3}$. Let us find the maximum projection of the slip velocity $f_{s m}$ on the axis $x_{3}$.

The averaged problem (16), (17) takes the form $\overline{\left(u_{1} u_{2}\right)} \rightarrow \max$

under conditions $\overline{u_{1}}=0, \overline{u_{2}}=0$.

Here the averaging is carried out on the set of admissible velocities $f^{k}, \quad k=1, \ldots, 4$.

The problem of choosing three basic values of the velocity vector from four reduces to a minimum in $\lambda$ from the maximum in $k$ of the Lagrange function

For $k=1,2,3,4$ :

$$
L=\sum_{k} f_{3}^{k}+\lambda_{1} \sum_{k} f_{1}^{k}+\lambda_{2} \sum_{k} f_{2}^{k} \rightarrow \min _{\lambda} \max _{k}
$$


Conditions for the Optimality of the Averaged Problem of Nonlinear Programming and Synthesis of the Philippov's Theorem on Speed of Motion of Systems with the Discontinued Right Part of Differential Equations

$$
\begin{aligned}
& L^{1}=1+\lambda_{1}+\lambda_{2}, \\
& L^{2}=-1+\lambda_{1}-\lambda_{2}, \\
& L^{3}=-1-\lambda_{1}+\lambda_{2}, \\
& L^{4}=1-\lambda_{1}-\lambda_{2} .
\end{aligned}
$$

We choose $\lambda_{1}$ и $\lambda_{2}$ from the condition that the maximum of the Lagrange function be equal at the base points $L^{*}=L^{1}=L^{2}=L^{4}$

From the first equation

$$
1+\lambda_{1}+\lambda_{2}=-1+\lambda_{1}-\lambda_{2}=1-\lambda_{1}-\lambda_{2}
$$

$$
2+2 \lambda_{2}=0 \rightarrow \lambda_{2}=-1
$$

From the second equation

$$
2 \lambda_{1}=1-\lambda_{1}+1 \rightarrow \lambda_{1}=1 \text {. }
$$

The value of $L^{*}=1$.

Similarly by condition

$$
\begin{gathered}
L^{1}=L^{3}=L^{4}, \\
1+\lambda_{1}+\lambda_{2}=-1-\lambda_{1}+\lambda_{2}=1-\lambda_{1}-\lambda_{2},
\end{gathered}
$$

we get

$$
\lambda_{1}=-1, \quad \lambda_{2}=1, \quad L^{*}=1
$$

By the condition $L^{2}=L^{3}=L^{4}$ we have

$$
-1+\lambda_{1}-\lambda_{2}=-1-\lambda_{1}+\lambda_{2}=1-\lambda_{1}-\lambda_{2} \text {, }
$$

whence

$$
\lambda_{1}=\lambda_{2}=-1, \quad L^{*}=-1 \text {. }
$$

Thus, of the four vectors $f^{k}$ the basic ones are three: $f^{1}, f^{2}$ и $f^{4}$ или $f^{1}, f^{3}, f^{4}$.

Maximum sliding speed along $x_{3}$

$$
f_{s m}=\mu_{1} f_{3}^{1}+\mu_{2} f_{3}^{2}+\mu_{4} f_{3}^{4}=\mu_{1}-\mu_{2}+\mu_{4} .
$$

In this case, the equations (23) for calculating weight factors will take the form:

$$
\begin{aligned}
& \mu_{1} f_{1}^{1}+\mu_{2} f_{1}^{2}+\mu_{4} f_{1}^{4}=\mu_{1}+\mu_{2}-\mu_{4}=0, \\
& \mu_{1} f_{2}^{1}+\mu_{2} f_{2}^{2}+\mu_{3} f_{2}^{4}=+\mu_{1}-\mu_{2}-\mu_{4}=0, \\
& \mu_{1}+\mu_{2}+\mu_{4}=1 .
\end{aligned}
$$

Whence $\mu_{1}=\mu_{4}=0,5, \mu_{2}=0$. After substituting the weight factors in (30) we obtain

$$
f_{s m}=1 \text {. }
$$

Similarly, for the minimum speed along the $x_{3}$ we get $f_{s m}=-1$, which coincides with the result of considering this example in [1]n the basis of geometric considerations.

B. A system in which the vectors of permissible velocities depend on the control. Consider a system characterized by the equations:

$$
\dot{x}_{1}=\left(\frac{u_{1}}{u_{2}}-1\right)\left(1+x_{1}^{2}\right), \quad \dot{x}_{2}=\left(u_{1}-u_{2}\right) .
$$


Conditions for the Optimality of the Averaged Problem of Nonlinear Programming and Synthesis of the Philippov's Theorem on Speed of Motion of Systems with the Discontinued Right Part of Differential Equations

The line of switching $s(x)=x_{1}$. Control of $u_{1}$ undergoes a break on the line of switching $x_{1}=0$ :

$$
u_{1}=u_{1}^{+} \quad \text { at } x_{1}<0, \quad u_{1}=u_{1}^{-} \text {at } x_{1}>0, \quad u_{1}^{+}>u_{1}^{-}>0,
$$

the vector $\gamma$ is directed along the slip line, i.e. on the axis $x_{2}\left(\gamma_{1}=0, \gamma_{2}=1\right)$.

In this problem, you first need to find the base values of the control vector, and then the corresponding weighting coefficients and the slip speed.

The averaged problem (16), (17) takes the form

$$
{\overline{u_{1}-u_{2}}}^{u} \rightarrow \max ,{\overline{u_{1}-u_{2}}}^{u} \rightarrow \max ,
$$

under conditions

$$
{\overline{\left(\frac{u_{1}}{u_{2}}-1\right)}}^{u}\left(1+x_{1}^{2}\right)=0 .
$$

The Lagrange function for each of the values $u_{1}$ has the form:

$$
\begin{aligned}
& L_{+}=u_{1}^{+}-u_{2}^{+}-\lambda\left(\frac{u_{1}^{+}}{u_{2}^{+}}-1\right)\left(1+x_{1}^{2}\right), \\
& L_{-}=u_{1}^{-}-u_{2}^{-}-\lambda\left(\frac{u_{1}^{-}}{u_{2}^{-}}-1\right)\left(1+x_{1}^{2}\right) .
\end{aligned}
$$

We find the base values $u_{2}^{+}, u_{2}^{-}$by the condition of the maximum of each of these expressions by $u_{2}$ as a functions of $\lambda$. We get:

$$
u_{2}^{+}=\sqrt{\left(1+x_{1}^{2}\right) \lambda u_{1}^{+}}, \quad u_{2}^{-}=\sqrt{\left(1+x_{1}^{2}\right) \lambda u_{1}^{-}} .
$$

We substitute these expressions in $L_{+}$и $L_{-}$. One of them grows with the growth of $\lambda$, and the other decreases. The minimum of the maximum of the Lagrange function corresponds to the equality $L_{+}$and $L_{-}$. From this equality we find

$$
\sqrt{\left(1+x_{1}^{2}\right) \lambda}=\frac{\sqrt{u_{1}^{+}}+\sqrt{u_{1}^{-}}}{2} .
$$

The basic values of the control vector, whose number is $m+1=2$ :

$$
u^{+}=\left[u_{1}^{+} ; \quad u_{2}^{+}=0,5 \sqrt{u_{1}^{+}}\left(\sqrt{u_{1}^{+}}+\sqrt{u_{1}^{-}}\right)\right]
$$

and

$$
u^{+}=\left[u_{1}^{-} ; \quad u_{2}^{-}=0,5 \sqrt{u_{1}^{-}}\left(\sqrt{u_{1}^{+}}+\sqrt{u_{1}^{-}}\right)\right] .
$$

After determining the basic values of the control vector, we find the weight factors in the same way as in the degenerate problem. Substituting the base velocities in the expression for the rate of change $x_{1}$ and averaging the resulting velocities with the coefficients $\mu_{1}$ и $\mu_{2}=1-\mu_{1}$ so that the average rate of change $x_{1}$ was zero, we get that $\mu_{1}=\mu_{2}=0,5$. Finally, after substitution the base velocities and the found weights in the expression for the rate of change $x_{2}$ we obtain the slip velocity along this axis.

C. Tracking relay system with feedback. In the industry, widely distributed systems with electric motors, controlled by starters, the signal of which takes one of two possible values. The relay elements themselves are covered by feedback. In this case, a sliding mode occurs in the system, in which system behaves as a linear system, and the characteristics of such a «'quasilinear»' system depend on the type of feedback. 
Conditions for the Optimality of the Averaged Problem of Nonlinear Programming and Synthesis of the Philippov's Theorem on Speed of Motion of Systems with the Discontinued Right Part of Differential Equations

Let $x(t)$ be the rotation angle of the motor shaft and $d x / d t$ be the signal at its input. The signal at the system input is $r(t)$, and the signal at the feedback output is $y(t)$. Equations of motion:

$$
\frac{d x}{d t}=f_{+}^{x}(u)=1-u^{2}+u, \quad \frac{d y}{d t}=f_{+}^{y}(u)=\frac{1}{T}\left(y+1-u^{2}+u\right)
$$

for $s(y, t)=(r(t)-y(t))>0$;

$$
\frac{d x}{d t}=f_{-}^{x}(u)=-\left(u^{2}+1++u\right), \quad \frac{d y}{d t}=f_{-}^{y}(u)=\frac{1}{T}\left(y-u^{2}-1-u\right)
$$

for $s(y, t)=(r(t)-y(t))<0$.

We choose the vector $\gamma$ so that its direction coincides with the axis $x$. Values of the Lagrange function for different signs of $s$

$$
L_{+}=f_{+}^{x}(u)+\lambda\left(\frac{d r}{d t}-f_{+}^{y}(u)\right)
$$

and

$$
L_{-}=f_{-}^{x}(u)+\lambda\left(\frac{d r}{d t}-f_{-}^{y}(u)\right)
$$

must be maximal by $u$ and equal to each other (they are minimal by $\lambda$ ), which leads to equalities for basic controls, and the factor $\lambda: u_{+}=0,5, u_{-}=-0,5, \lambda=T$. For weighting coefficients we have equation

$$
\mu\left(\frac{d r}{d t}-f_{+}^{y}(u)\right)+(1-\mu)\left(\frac{d r}{d t}-f_{-}^{y}(u)\right)=0,
$$

whence $\mu(t, y)=0,75-y+T \frac{d r}{d t}$, taking the boundary values ??when the right-hand side of the equation is greater than one or less than zero.

In sliding mode, the system obeys the equations:

$$
\begin{gathered}
\dot{x}_{s m}=\mu f_{+}^{x}(u)+(1-\mu) f_{-}^{x}(u)=-\left(y+T \frac{d r}{d t}\right), \\
\dot{y}_{s m}=\mu f_{+}^{y}(u)+(1-\mu) f_{-}^{y}(u)=-\frac{d r}{d t} .
\end{gathered}
$$

After excluding $\frac{d r}{d t}$ we get the connection between $x_{s}$ и $y_{s}$ in the form: $\dot{x}_{s m}=-\left(y_{s}+T \dot{y}_{s m}\right)$, so the system behaves as linear in the sliding mode.

\section{DiscusSion}

In an equivalent control's method is proposed to determine $f_{s m}(x, t)$ according to which the slip velocity vector is found after substitution of the equivalent control $u_{e q}$ in the equations of motion, so that $f_{s m}(x, t)=f\left(x, t, u_{e q}\right)$. The equivalent control for each $t, x \in S$ is determined from the conditions

$$
\varphi_{i}\left(x, t, u_{e q}\right)=0, \quad i=1, \ldots, m,
$$

where $\varphi_{-}$i is defined by the equality (14)

For systems that are affine in control, in particular for an important class of systems with variable structure, the method of equivalent control leads to the same results as the averaging of the permissible velocities according to A.F. Filippov if the system of linear equations for $u_{e q}$ has a unique solution. All the difficulties that can arise when solving this system are discussed in detail in [2].

When the right-hand sides of the equations of motion are nonlinear in control, the functions $f_{s m}(x, t)$, found by the method of averaging admissible velocities and the equivalent control method are different (see the example in [1]).

In the general case, the velocities of the system can be undetermined outside the set $V_{u}$ of admissible controls. From their additional definition, the equivalent control, and hence the slip speed found from it, depends. In the 
Conditions for the Optimality of the Averaged Problem of Nonlinear Programming and Synthesis of the Philippov's Theorem on Speed of Motion of Systems with the Discontinued Right Part of Differential Equations

method of averaging the velocities, it depends only on the admissible base velocities. In addition, the equations (36) may not have a real solution for all or for some $x, t$. For example, the dimension of the vector of controls can be less than $m$.

In a number of cases, among the admissible velocities, a velocity directed along the slip surface [9] can appear. It summarized with each of the other permissible speeds, and therefore with the speed of sliding, found as it was already mentioned.

The determination of the slip velocity as an average velocity of the system, obtained under the condition that the average rate of change of all functions $s_{i}(x)$, defining the surface of switching is zero, allows us to determine in which cases the slip velocity can be is uniquely determined and how its values can be obtained, by purely formal procedures of the averaged optimization. The solution of the problem does not depend on whether the discontinuity of the right-hand sides of the equation is a consequence of a discontinuity of the control or a change in the form of the dependence $f(x, u, t)$ under the change of the sign $s$.

The authors is grateful to V.I. Utkin for a useful discussion of the article.

\section{REFERENCES}

1. Utkin V.I. Short Commentary on the Method of Continuation of Solution on the Discontinuity Boundary. Autom. Remote Control, 2015, 5:165-174.

2. Utkin V.I. Sliding modes and their applications in systems with variable structure. Nauka Publishers, 1974.

3. Filippov A.F. Differential equations with discontinuous right-hand side. Mat. compilation, 1960, 99-128.

4. Utkin V.I. Sliding modes in optimization and control tasks. Nauka Publishers, 1981.

5. Neimark Yu.I. About sliding mode of relay systems of automatic regulation. Autom. Remote Control, 1957, $1: 27-33$.

6. Tsypkin Ya.Z. The theory of automatic control systems. State Publishing House of Technical and Theoretical Literature, 1955.

7. Tsirlin A.M. The methods of averaged optimization and their applications. PhyzMatLit, 1977.

8. Rozonoer L.I., Tsirlin A.M. Optimal control of thermodynamic systems. Autom. Remote Control, 1983, 1-3:70-79.

9. Jefrrey M.R. Dynamics at a Switcing Intersection Hierarchy. Physica D, 2014, 1:34-45.

Citation: Margarita A. ZAEVA, Anatoly M. TSIRLIN. "Conditions for the Optimality of the Averaged Problem of Nonlinear Programming and Synthesis of the Philippov's Theorem on Speed of Motion of Systems with the Discontinued Right Part of Differential Equations". American Research Journal of Mathematics. vol 3, no. 1, 2017, pp. 1-11.

Copyright (C) 2017 Margarita A. ZAEVA, Anatoly M. TSIRLIN, This is an open access article distributed under the Creative Commons Attribution License, which permits unrestricted use, distribution, and reproduction in any medium, provided the original work is properly cited. 